\title{
REGULAR NORMAL EXTENSIONS OVER COMPLETE FIELDS*
}

\author{
BY \\ O. F. G. SCHILLING
}

The local class field theory completely settles all problems pertaining to the abelian extensions of a complete field. All abelian extensions can be described in terms of norm class groups and it can readily be decided which abelian groups can be realized as Galois groups. In this paper we want to attack a more general problem. We consider non-abelian normal extensions of the ground field whose degrees are powers of a prime which is distinct from the characteristic of the residue class field. We construct an infinite normal extension which acts as an universal field. The algebraic approximation of this field yields complete information on the algebraic structure of the field and its Galois group over the ground field. It is shown that the Galois group contains an everywhere dense subgroup which completely suffices to describe the Galois theory. The structure of this group is readily determined. To a certain extent it is a generalization of the fuchsian groups of the classical theory of algebraic functions. The analogy is rather striking. We thus can associate to the given rational prime and the ground field an abstract infinite discrete group which can be considered as a universal covering group with respect to the given prime. All finite groups which can be realized as Galois groups are homomorphic maps of this infinite group. Finally, we associate to certain non-abelian extensions factor groups which are defined in terms of division algebras over the ground field. These factor groups are isomorphic with the respective Galois groups of the fields under consideration. We thus obtain another generalization of local class field theory. In our proofs we make ample use of the ramification theory and local class field theory.

Let $k$ be a field which is complete with respect to a discrete valuation of rank one. Suppose that $\mathfrak{o}$ is the ring of all integers in $k$ and that $\mathfrak{l}=(\lambda)$ is the prime ideal of $\boldsymbol{o}$. We shall assume that the residue field $\mathfrak{o} / \mathfrak{l}$ of $k$ is a finite Galois field containing $l^{\nu}=q$ elements. Let $p \neq 2$ be a prime such that $q-1 \equiv 0$ $(\bmod p)$. Then $k$ contains the $p$ th roots of unity. $\dagger$ In the sequel we shall sup-

* Presented to the Society, October 28, 1939; received by the editors January 31, 1940. This paper was received by the editors of the Annals of Mathematics August 15, 1939, accepted by them, and later transferred to these Transactions.

$\dagger$ Since $k$ is supposed to be complete, Hensel's irreducibility criterion implies the existence of the $(q-1)$ st roots of unity in $k$. Cf. [4]. The number [4] refers to the reference in the bibliography at the end of this paper. 
pose once and for all that $k$ and $p$ are fixed. The structure theory of complete fields yields that $k$ is either a field of $\mathfrak{l}$-adic numbers over the rational $l$-adic field or a field of formal power series in one variable over the Galois field of $q$ elements (cf. [7, 11, 12.2]).

Lemma 1. The maximal abelian extension $A$ of exponent pover $k$ has a Galois group of type $(p, p)$. The field $A$ contains the unramified extension $U$ of degree $p$ over $k$.

Proof. $\dagger$ The field $A$ is the join of all radical extensions $k\left(a^{1 / p}\right), a \neq 0$ in $k$, for $k$ contains the $p$ th roots of unity. Hence, by the general theory of radical extensions, we must investigate the structure of the factor group $k^{*} / k^{* p}$, where $k^{*}$ denotes the multiplicative group of the field $k$. Let $\{\epsilon\}$ be the group of all units in $k$ which are congruent to $1(\bmod \mathfrak{l})$. We first assert that $\{\epsilon\}=\left\{\epsilon^{p}\right\}$, that is, every unit $\epsilon$ is the pth power of a suitable unit $\eta \varepsilon\{\epsilon\}$. We shall construct a solution $\eta$ of $x^{p}-\epsilon=0$ by successive approximation. Since $\epsilon \equiv 1(\bmod \mathfrak{l})$, we can put $\eta_{1}=1$. Suppose that we already constructed a unit $\eta_{i}$ such that $\epsilon \equiv \eta_{i}^{p}\left(\bmod \mathfrak{\complement}^{i+1}\right)$. We set $\eta_{i+1}=\eta_{i}+x_{i+1} \lambda^{i+1}$ where $x_{i}$ is to be determined in a fixed set of representatives for $\mathfrak{o} / \mathfrak{l}$. We require

$$
\epsilon \equiv \eta_{i+1}^{p}\left(\bmod \mathfrak{l}^{i+2}\right) .
$$

Consequently, we have $\epsilon \equiv\left(\eta_{i}+x_{i+1} \lambda^{i+1}\right)^{p}\left(\bmod \mathfrak{l}^{i+2}\right)$, or $\epsilon \equiv \eta_{i}^{p}+p \eta_{i}^{p-1} x_{i+1} \lambda^{i+1}$ $\left(\bmod \mathfrak{I}^{i+2}\right)$; thus

$$
\left(\epsilon-\eta_{i}^{p}\right)\left(p \eta_{i}^{p-1}\right)^{-1} \equiv x_{i+1} \lambda^{i+1}\left(\bmod \mathfrak{l}^{i+2}\right),
$$

for $(p, \mathfrak{l})=1$ by assumption. Hence

$$
\left(\epsilon-\eta_{i}^{p}\right)\left(p \eta_{i}^{p-1}\right)^{-1} \lambda^{-(i+1)} \equiv x_{i+1}(\bmod \mathfrak{l}),
$$

that is, $x_{i+1}$ is uniquely determined by $\epsilon$. Thus $k^{* p}$ contains the group $\{\epsilon\}$. Since $k$ is a complete field, every element $a \varepsilon k$ has a unique representation as $\omega^{\alpha} \lambda^{\beta} \epsilon$ where $\omega$ denotes a fixed primitive $(q-1)$ st root of unity and $\lambda$ a fixed prime element of $k$. Hence $k^{*}\left(\bmod k^{* p}\right)$ has $\omega$ and $\lambda$ as independent generating representatives. Since $q-1 \equiv 0(\bmod p)$, the group $k^{*} / k^{*} p$ has type $(p, p)$. Consequently, the field $A$ is given as $k\left(\lambda^{1 / p}, \omega^{1 / p}\right)$. Obviously, the cyclic unramified field $U$ of degree $p$ over $k$ is contained in $A$. This completes the proof of the lemma.

Suppose that $p^{\mu}$ is the highest power of $p$ which divides $q-1$. Then the maximal abelian extension of exponent $p^{\mu}$ has degree $p^{2 \mu}$ and its Galois group has type $\left(p^{\mu}, p^{\mu}\right)$. Now let $i>\mu$. We want to find the order of the factor group

$\dagger$ We repeat here Hensel's arguments for sake of completeness. Cf. [5.1]. 
$k^{*} / k^{*} p^{i}$. Obviously the prime element $\lambda$ is a representative of order $p^{i}$. Consider now the factor group $\{\omega\} /\left\{\omega^{p^{i}}\right\}$. We find for its order

$$
\begin{aligned}
& {\left[\{\omega\}:\left\{\omega^{p^{i}}\right\}\right]=\left[\{\omega\}:\left\{\omega^{p}\right\}\right]} \\
& \cdots\left[\left\{\omega^{\mu}\right\}:\left\{\omega^{\mu+1}\right\}\right]\left[\left\{\omega^{\mu+1}\right\}:\left\{\omega^{\mu+2}\right\}\right] \cdots\left[\left\{\omega^{p^{i-1}}\right\}:\left\{\omega^{p^{i}}\right\}\right] .
\end{aligned}
$$

Hence by the index principle of group theory (considering $\omega \rightarrow \omega^{p}$ as a homomorphism), $\left[\{\omega\}:\left\{\omega^{p^{\mu}}\right\}\right]=\left[\{\omega\}:\left\{\omega^{p^{i}}\right\}\right]=p^{\mu}$ for $p^{\mu} \| q-1$. Thus, we have

Lemma 2. The index $\left[k^{*}: k^{*} p^{i}\right]=p^{i+\mu}$ if $i>\mu$ and $p^{2 i}$ if $i \leqq \mu$.

Suppose now that $K$ is an arbitrary normal extension of $k$ whose degree is a power of $p$. Let $G=\{x, y, \cdots\}$ be the Galois group of $K / k$. The elements $x y x^{-1} y^{p-1}$ generate an invarant subgroup $G^{*}$ of $G$ whose factor group $G / G^{*}$ has type $(p, p, \cdots, p)$ (cf. [12.1]). Let $K^{*}$ be the field which corresponds to $G^{*}$.

THEOREM 1. The Galois group of any normal extension $K$ of degree $p^{n}$ over $k$ can always be generated by two elements.

Proof. Let $G^{*}$ be the group $\left\{x y x^{-1} y^{p-1}\right\}$. Then the minimal number of generators of $G$ is equal to the number of invariants of $G / G^{*}$. This number of invariants is not greater than 2 for $\left[K^{*}: k\right]=\left[G: G^{*}\right] \leqq[A: k]=p^{2}$ by Lemma 1.

A normal field $K$ over $k$ of degree $p^{n}$ shall be called a regular extension of $k$.

We now want to construct a universal field $N^{(p)}$ over $k$ which contains all regular extensions $K$. Let $A=A_{1}$ be the maximal abelian extension of exponent $p$ over $k$. Since $k \subset A_{1}$, that is, the $p$ th roots of unity lie in $A_{1}$, we can repeat this construction. Let $k \subset A_{1} \subset A_{2} \subset \cdots \subset A_{i-1} \subset A_{i} \subset \cdots$ be the infinite tower of relative maximal abelian extensions of exponent $p$; that is, $\left[A_{i}: A_{i-1}\right]=p^{2}$, the Galois groups $G\left(A_{i}, A_{i-1}\right)$ having type $(p, p)$. A theorem of local class field theory yields that $A_{i}$ is normal over $k$, the degree being $p^{2 i}$. Namely, the class group $A_{i-1}^{* p}$ which belongs to $A_{i} / A_{i-1}$ is left invariant by all elements of $G\left(A_{i-1}, k\right)$ (cf. [3]). Consequently, the join $N^{(p)}=\sum A_{i}$ is an infinite normal extension of $k$.

THEOREM 2. The field $N^{(p)} / k$ is universal, that is, it contains all regular fields $K / k$.

Proof. The Galois group $G$ of $K / k$ contains a series of normal subgroups $\bar{G}_{i}$ such that $\bar{G}_{i} / \bar{G}_{i+1}$ are cyclic groups of order $p$. Let

$$
k \subset K_{1} \subset K_{2} \subset \cdots \subset K_{i} \subset \cdots \subset K
$$

be the associated chain of subfields (cf. [12.1]). In order to prove our assertion we compare this chain with the defining chain $k \subset A_{1} \subset A_{2} \subset \cdots$ of $N^{(p)}$. 
We have $K_{1} \subset A_{1}$, for $K_{1}$ is a cyclic extension of degree $p$ over $k$. Consider now the cyclic extension $K_{2}$ of $K_{1}$. Then either $K_{2}=A_{1}$ or $K_{2} \neq A_{1}$. In the second case the join $K_{2} A_{1}$ is a cyclic extension of degree $p$ over $A$, as a consequence of the Galois theory. Consequently $K_{2} A_{1} \subset A_{2}$ by construction of $A_{2}$. We can continue this process. Thus, ultimately $K \subset A_{j}, \$ A_{j-1}$ where the index $j$ is uniquely determined by the given field $K$.

An immediate consequence of Theorem 2 is the fact that the Galois group $G$ of a regular field $K$ is a homomorphic map $G^{(p)} / S(K)$ of the Galois group $G^{(p)}$ belonging to the universal field $N^{(p)} / k$. We therefore must investigate the structure of $G^{(p)}$ if we want to get information on the various groups $G$.

Let $G_{i}$ be the Galois group of an arbitrary but fixed extension $A_{i}$ over $k$. Then Theorem 1 implies that $G_{i}$ can be generated by 2 elements $\sigma_{i}, \tau_{i}$. We shall select $\sigma_{i}, \tau_{i}$ such that $\sigma_{i}$ generates the inertial group of $A_{i}$ with respect to $k$. The group $\left\{\sigma_{i}\right\}$ is an invariant subgroup of $G_{i}$ and its order is equal to $p^{i}$, for $A_{i}$ contains the unramified field $U_{p}$ (of degree $p^{i}$ over $k$ ) as maximal unramified subfield (inertial field) (cf. [4]). Thus

$$
G_{i} /\left\{\sigma_{i}\right\}=G\left(U_{p i}, k\right) .
$$

The group $G\left(U_{p^{i}}, k\right)$ has order $p^{i}$ and is generated by an element $\tau_{i}^{*}$. Thus, if $\tau_{i}$ denotes a representative of $\tau_{i}^{*}$ in $G_{i}$, we get

$$
\left\{\sigma_{i}, \tau_{i}\right\}=G_{i}, \quad \sigma_{i}^{p i}=\tau_{i}^{p i}=1, \quad \tau_{i}^{-1} \sigma_{i} \tau_{i}=\sigma_{i}^{o_{2}}
$$

where $g_{i}$ is a prime residue modulo $p^{i}$. The group $G_{i-1}$ is a homomorphic map of $G_{i}$. Let $S_{i}$ denote the invariant subgroup of $G_{i}$ which belongs to $A_{i-1}$. Then $G_{i} / S_{i} \cong G_{i-1}$. It is immediately seen, as a consequence of our selection of the generators $\sigma_{i}, \tau_{i}$, that $S_{i}=\left\{\sigma_{i}^{p^{i-1}}, \tau_{i}^{p^{i-1}}\right\}$. Hence we can select the generators $\sigma_{i-1}, \tau_{i-1}$ as the maps of $\sigma_{i}, \tau_{i}$ on $G_{i-1}$. Whence $\tau_{i-1}^{-1} \sigma_{i-1} \tau_{i-1}=\sigma_{i-1}^{\sigma_{i}}=\sigma_{i-1}^{\sigma_{i-1}}$ and consequently $g_{i} \equiv g_{i-1}\left(\bmod p^{i-1}\right)$.

Next we want to normalize the exponents $g_{i}$ to an integer $g$ such that $\tau_{i}^{-1} \sigma_{i} \tau_{i}=\sigma_{i}^{o}$ for all $i$. We shall prove this statement by constructing two elements $\sigma, \tau$ in the infinite Galois group of $N^{(p)} / k$ whose homomorphic images in $G_{i}$ have the required properties.

Suppose that $p$ is an odd rational prime. Denote by $V$ the valuation which is given by $p$.

LEMмA 3. Let a be a p-adic integer such that $p^{\mu} \| a-1, \mu \geqq 1$; then there exists $a$-adic integer $h$ such that $a^{h}=1+p^{\mu}$. In other words, the p-adic units which are congruent to $1\left(\bmod p^{\mu}\right)$ form an ideal cyclic group which is generated by any $a \equiv 1\left(p^{\mu}\right), a \neq 1\left(p^{\mu+1}\right)$.

Proof. The exponent $h$ for which $a^{h}=1+p^{\mu}$ will be constructed by suc- 
cessive $p$-adic approximation. Let $a=1+b p^{\mu}$ where $b$ is a $p$-adic integer of value 0 . Suppose that we already found integers $h_{1}, \cdots, h_{j}$ for which

$$
h_{\nu} \equiv h_{\nu-1}\left(\bmod p^{\nu-1}\right)
$$$$
\nu=1,2, \cdots, j,
$$

and

$$
a^{h_{\nu}} \equiv 1+p^{\mu}\left(\bmod p^{\mu+\nu}\right), \quad \nu=1,2, \cdots, j .
$$

The first number $h_{1}$ is easily determined. Namely, we require

$$
a^{h_{1}}=\left(1+b p^{\mu}\right)^{h_{1}}=1+h_{1} b p^{\mu}+p^{2 \mu} c, \quad V(c) \geqq 0 .
$$

Since $b \neq \equiv 0(\bmod p)$, we can determine $h_{1}$ by the congruence $h_{1} b \equiv 1(\bmod p)$. Consequently,

$$
a^{h_{1}} \equiv 1+p^{\mu}\left(\bmod p^{\mu+1}\right) .
$$

In the general case we propose to find an integer $h_{j+1}$ as $h_{j}+r_{j} p^{j}$ where $r_{j}$ has to be determined. Such a number $h_{j+1}$ surely satisfies condition (1). In order to show that condition (2) can be realized we proceed as follows. We must have

$$
a^{h_{j+1}}=a^{h_{j}} a^{p^{r_{j}}}=\left(1+p^{\mu}+s p^{\mu+j}\right)\left(1+b p^{\mu}\right)^{r_{j} p}
$$

where $s$ is a $p$-adic integer by induction. Next

$$
a^{r_{j} p^{j}}=\left(1+b p^{\mu}\right)^{r_{j} p^{i}}=1+r_{j} b p^{\mu+j}+\sum_{\kappa=2}^{r_{j} p^{j}} C_{r_{j} p^{i, \kappa}}\left(b p^{\mu}\right)^{\kappa} .
$$

We now show that the value of the sum on the right hand exceeds $\mu+j$. The binomial coefficients $C_{r_{j} p^{j}, \kappa}=B_{j}$ have the form $p^{i} r_{j}(\kappa !)^{-1} d$ where $V(d) \geqq 0$. Hence

$$
V\left(B_{j}\right) \geqq j-V(\kappa !) \geqq j-\kappa(p-1)^{-1},
$$

for $V(\kappa !)=\left(\kappa-s_{\kappa}\right)(p-1)^{-1}$ where $s_{\kappa} \geqq 0$ (cf. [5.2]). Thus

$$
\begin{aligned}
V\left(B_{j}\left(b p^{\mu}\right)^{\kappa}\right) & \geqq \mu \kappa+j-\kappa(p-1)^{-1}=\mu+j+\mu(\kappa-1)-\kappa(p-1)^{-1} \\
& \geqq \mu+j+\mu(\kappa-1)-\kappa / 2 \geqq \mu+j+(\kappa-1)-\kappa / 2>\mu+j,
\end{aligned}
$$

provided that $\mu \geqq 1, p \geqq 3, \kappa \geqq 3$. If $\kappa=2$, then

$$
B_{j}\left(b p^{\mu}\right)^{2}=r_{j} p^{i}\left(r_{j} p^{i}-1\right) 2^{-1} b^{2} p^{2 \mu}=p^{2 \mu+j} d,
$$

where $d$ is a $p$-adic integer. Consequently,

$$
V\left(B_{j}\left(b p^{\mu}\right)^{2}\right)=2 \mu+j \geqq \mu+j+1 .
$$

Hence, in general, $a^{p^{j} r_{j}}=1+b r_{j} p^{\mu+j}+t p^{\mu+j+1}$, where $V(t) \geqq 0$. Returning to $a^{h_{j+1}}$ we get 


$$
\begin{aligned}
a^{h+1} & =\left(1+p^{\mu}+s p^{\mu+j}\right)\left(1+b r_{j} p^{\mu+j}+t p^{\mu+j+1}\right) \\
& \equiv 1+p^{\mu}+\left(s+b r_{j}\right) p^{\mu+j}\left(\bmod p^{\mu+j+1}\right) .
\end{aligned}
$$

Since $b \neq \equiv 0(\bmod p)$, we can determine $r_{j}$ as a solution of $s+b r_{j} \equiv 0(\bmod p)$. Hence the induction is completed. The numbers $h_{j}$ form a convergent $p$-adic sequence. Let $\lim _{j \rightarrow \infty} h_{j}=h$. Then

$$
a^{h}=a^{1 \mathrm{im} h_{j}}=\lim _{j \rightarrow \infty} a^{h_{j}}=1+p^{\mu} .
$$

Lemma 4. The Galois group $G_{i}$ of $A_{i} / k$ is given by the relations $\sigma_{i}^{p^{i}}=\tau_{i}^{p^{i}}=1$, $\tau_{i}^{-1} \sigma_{i} \tau_{i}=\sigma_{i}^{1+p^{\mu}}$ where $a-1=p^{\mu} \cdot r,(r, p)=1$.

Proof. Let $G^{(p)}$ be the infinite Galois group of $N^{(p)} / k$. As usual we define $G^{(p)}$ as the group of vectors $\left(\rho_{1}, \cdots, \rho_{i}, \cdots\right)$ where (cf. [6])

$$
\rho_{i} \varepsilon G_{i}, \rho_{i}\left(\bmod S_{i}\right)=\rho_{i-1}, \quad i=1,2, \cdots .
$$

Application of the ramification theory of infinite normal extensions yields that $G^{(p)}$ contains two elements $\sigma, \tau^{*}$ having the following properties. The element $\sigma$ is a generator of the inertial group of $N^{(p)} / k$, that is, the elements of the inertial group are powers $\sigma^{c}$ where the $c$ 's are $p$-adic integers. The group of the inertial field of $N^{(p)} / k$ is ideal cyclic and generated by a residue class $\tau^{*}(\bmod \{\sigma\})$ where $\tau_{\varepsilon}^{*} G^{(p)}$ (cf. [6]). Since the inertial group is an invariant subgroup of $G^{(p)}$, we have $\tau^{*-1} \sigma^{*} \tau^{*}=\sigma^{a}$ where $a$ is a $p$-adic integer. Since $\lim _{i \rightarrow \infty} G_{i}=N^{(p)}$, we have $\sigma=\left(\sigma_{1}, \cdots, \sigma_{i}, \cdots\right), \sigma_{i} \varepsilon G_{i}$, and $\tau^{*}=\left(\tau_{1}^{*}, \cdots, \tau_{i}^{*}, \cdots\right), \tau_{i}^{*} \varepsilon G_{i}$. Moreover, $\tau_{i}^{*-1} \sigma_{i} \tau_{i}^{*}=\sigma_{i}^{a_{i}}$ where $a_{i} \equiv a(\bmod$ $\left.p^{i}\right)$ and $\sigma_{i}^{p_{i}}=\tau_{i}^{*} p^{i}=1$ according to the selection of the elements $\sigma, \tau^{*}$ in $G^{(p)}$.

We now want to apply Lemma 3 in order to normalize the exponents $g_{i}$. Consider for this purpose the norm groups $N_{i} A_{i}^{*}$ which belong to the various fields $A_{i} / k ; N_{i}$ denotes the norm taken from $A_{i}$ to $k$. Observing that $N_{i}$ can be split up into the various relative norms from $A_{j}$ to $A_{j-1}$ and that $A_{j}$ is the maximal abelian extension of type $(p, p)$ over $A_{j-1}$, we get $N_{i} A_{i}^{*}=k^{*} p^{i}$. Hence

$$
\begin{aligned}
\left.\mathfrak{l}^{*}: N_{i} A_{i}^{*}\right] & =p^{2 i} \quad \text { if } \quad i \leqq \mu \\
& =p^{\mu+i} \text { if } \quad i>\mu .
\end{aligned}
$$

A theorem of local class field theory yields $\dagger$ that

(i) $A_{i}$ is an abelian extension of type $\left(p^{i}, p^{i}\right)$ over $k$ if $i \leqq \mu$,

(ii) $A_{i}$ is a non-abelian extension of degree $p^{2 i}$ over $k$ if $i>\mu$ and the group $N_{i} A_{i}{ }^{*}$ belongs to the maximal abelian subfield $A_{\mu} U_{p^{i}}$ of $A_{i}$.

Since $G_{\mu}$ is the last Galois group in the approximation of $G^{(p)}$ which is abelian, we must have $a \equiv 1\left(\bmod p^{\mu}\right), a \neq \equiv 1\left(\bmod p^{\mu+1}\right)$. Hence there exists,

$\dagger[3,10]$. We mean the "Abgrenzungssatz" of local class field theory. 
by Lemma 3 , a $p$-adic integer $h$ such that $a^{h}=1+p^{\mu}$. Consequently, we can change $\tau^{*}$ to a new element $\tau=\tau^{*_{h}}$ in $G^{(p)}$ such that

$$
\tau^{-1} \sigma \tau=\sigma^{1+p^{\mu}} \text {. }
$$

Namely, $\tau^{*-1} \sigma \tau^{*}=\sigma^{a}$ implies $\tau^{*-h} \sigma \tau^{* h}=\sigma^{a h}=\sigma^{1+p^{\mu}}$. Hence, also $\tau_{i}^{-1} \sigma_{i} \tau_{i}=\sigma_{i}^{1+p^{\mu}}$, $i=1,2, \cdots$.

Lemma 5. Let $L$ be an infinite normal extension of the abstract field $R$ and let $\Gamma$ be the (topologized) Galois group of $L / R$. Suppose that $\Delta$ is a subgroup of $\Gamma$. Then $\Delta$ is everywhere dense in $\Gamma$ if and only if whenever $W / R$ is a finite normal subfield of $L$ every automorphism of $W / R$ can be extended to an automorphism of $\Delta . \dagger$

Proof. We first note that a group $\Delta$ as described in the lemma is sufficient to describe the Galois theory of the finite extensions $W / R$ which lie in $L$. An alternate formulation of the conditions of the lemma is this:

If $L \supset W \supset R,[W: R]$ finite, and if $\Delta(W)$ is the subgroup of $\Delta$ leaving the elements of $W$ fixed, then $\Delta / \Delta(W)$ is isomorphic to $G(W, R)$.

Suppose now that $\Delta$ is an everywhere dense subgroup of $\Gamma$. We want to prove that the Galois theory for finite extensions $R \subset W \subset L$ can be described in terms of $\Delta$. Let $\rho$ be an arbitrary automorphism of $G(W, R)$. We can extend $\rho$ to an automorphism $\rho^{\prime}$ of $\Gamma$. Since $\Delta$ is everywhere dense in $\Gamma$ and since the automorphisms leaving $W$ elementwise fixed constitute a neighborhood $N$ of the unit in $\Gamma$, there exists an element $\delta$ in $\Delta$ such that $\delta \rho^{\prime-1}$ lies in $N$. Thus, $\delta$ lies in $N \rho^{\prime}$ or $\delta$ induces $\rho$ on $W$. Conversely, the possibility of describing the Galois theory for finite extensions $W / R$ by means of a subgroup $\Delta$ of $\Gamma$ implies that $\Delta$ is everywhere dense in $\Gamma$. Let $\rho^{\prime}$ be any automorphism of $\Gamma$ and let $N(W)$ be any neighborhood of the unit in $\Gamma$, that is, $N(W)$ is defined by a finite subfield $W / R$ of $L$. Then there exists, by hypothesis, an element $\delta$ in $\Delta$ such that $\delta$ agrees with $\rho^{\prime}$ on $W$, that is, $\delta \rho^{\prime-1}$ induces the identity on $W$. Hence $\delta \rho^{\prime-1}$ is an element of $N(W)$. This means that $\Delta$ is an everywhere dense subgroup of $\Gamma$.

Theorem 3. The group $\left\{\sigma, \tau ; \tau^{-1} \sigma \tau=\sigma^{1+p^{\mu}}\right\}=F^{(p)}$ is an everywhere dense subgroup of $G^{(p)}$. The Galois theory for finite subfields of $N^{(p)} / k$ can be described in terms of the subgroup $F^{(p)}$.

Proof. By Lemma 5 it suffices to prove the first part of the theorem. The topology of $G^{(p)}$ is given by the chain of homomorphisms $G_{i} \rightarrow G_{i-1}$, explicitly

$$
\sigma_{i-1}=\sigma_{i}\left(\bmod \left\{\sigma_{i}^{p^{i-1}}, \tau_{i}^{p^{i-1}}\right\}\right), \quad \tau_{i-1}=\tau_{i}\left(\bmod \left\{\sigma_{i}^{p^{i-1}}, \tau_{i}^{p^{i-1}}\right\}\right) .
$$

† The author wants to thank Professor Saunders MacLane for valuable suggestions in the proofs of Lemmas 3, 5. 
In other words, we get an isomorphic representation of $G_{i-1}$ by reducing the exponents of the elements $\rho_{i}=\sigma_{i}^{\alpha} \tau_{i}^{\beta}$ in $G_{i}$ modulo $p^{i-1}$ and changing the subscript $i$ to $i-1$. Since $\sigma_{i}^{p^{i}}=\tau_{i}^{p^{i}}=1$, the exponents $\alpha, \beta$ of the element $\rho_{i} \varepsilon G_{i}$ are $p$-adic integers reduced modulo $p^{i}$. Next we remark that $\left\{\sigma^{p^{i}}, \tau^{p^{i}}\right\}$ is an invariant subgroup $F_{i}$ of $F^{(p)}$. Namely, we have

$$
\begin{aligned}
\tau^{-1} \sigma^{p^{i}} \tau & =\tau^{-1} \sigma \tau \tau^{-1} \sigma \tau \cdots \tau^{-1} \sigma \tau \\
& =\sigma^{1+p^{\mu}} \sigma^{1+p^{\mu}} \cdots \sigma^{1+p^{\mu}} \\
& =\sigma^{\left(1+p^{\mu}\right) p^{i}}=\left(\sigma^{p^{i}}\right)^{1+p^{\mu}} \varepsilon\left\{\sigma^{p^{i}}, \tau^{p^{i}}\right\} .
\end{aligned}
$$

Next $\sigma \tau \sigma^{-1}=\tau \sigma^{p^{\mu}}$; hence

$$
\sigma \tau^{p^{i} \sigma^{-1}}=\sigma \tau \sigma^{-1} \sigma \tau \sigma^{-1} \cdots \sigma \tau \sigma^{-1}=\tau \sigma^{p^{\mu}} \tau \sigma^{p^{\mu}} \cdots \tau \sigma^{p^{\mu}}=\tau^{p^{i}}\left(\sigma^{p^{i}}\right)^{x}
$$

where $x=\left[\left(1+p^{\mu}\right)^{p^{i}-1}\right] p^{-i}$. Thus $F^{(p)} / F_{i} \cong G_{i}$. In other words, we obtain $G_{i}$ from $F^{(p)}$ by reducing the exponents $\alpha, \beta$ of $\sigma^{\alpha} \tau^{\beta}$ ( $\alpha, \beta$ are rational integers) modulo $p^{i}$ and attaching the subscript $i$ to $\sigma, \tau$. Consequently, every element $\rho=\left(\rho_{1}, \cdots, \rho_{i}, \cdots\right)$ of $G^{(p)}$ can be approximated by a sequence $\rho^{(i)}=\sigma^{\alpha(i)} \tau^{\beta(i)}$, $i=1,2, \cdots$, where the $\alpha(i), \beta(i)$ are integers such that

$$
\rho_{i}=\rho^{(i)}\left(\bmod S^{(i)}\right)=\sigma^{\alpha(i)} \tau^{\beta(i)}\left(\bmod F^{(i)}\right)
$$

for every $i$, where $G^{(p)} / S^{(i)} \cong G_{i}$. This proves that $F^{(p)}$ is an everywhere dense subgroup of $G^{(p)}$. We remark that the closure $G^{(p)}$ of $F^{(p)}$ is obtained by admitting for the exponents $\alpha, \beta$ of $\rho=\sigma^{\alpha} \tau^{\beta}$ arbitrary $p$-adic integers instead of rational integers. The closure of $F_{i}$ in $G^{(p)}$ is equal to $S^{(i)}$ and $S^{(i)} \cap F^{(p)}=F_{i}$. This follows immediately from the imbedding of $F^{(p)}$ in $G^{(p)}$.

Theorem 4. A finite group $G$ of order $p^{n}$ can be realized as the Galois group of a reguar extension $K / k$ if and only if it is a homomorphic map of the group $F^{(p)}=\left\{\sigma, \tau ; \tau^{-1} \sigma \tau=\sigma^{1+p^{\mu}}\right\}$.

Proof. Let $K$ be an arbitrary regular extension of $k$. Then $K \subseteq A_{i}, i$ sufficiently large, by Theorem 2. Then, by Theorem 3, the Galois group $G$ of $K$ is a homomorphic map of $F^{(p)}$. Conversely, suppose that $G \cong F^{(p)} / T$. Then, by the second half of Theorem 3 , there exists at least one field $K \supset k$ whose Galois group is isomorphic with $G$. The field $K$ belongs to $T$. However, $T$ need not be uniquely determined by $G$, for the homomorphism $F^{(p)} \rightarrow G$ can, in general, be realized by various invariant subgroups $T$. The number of groups $T$ corresponding to a given group $G$ is finite. Namely, the argument which we used to prove that $K \subset A_{i}$ shows that $i$ is bounded by $n$, where $[K: k]=p^{n}$. Hence $G \cong G(K, k)=G_{i} / G\left(A_{i}, K\right)=\left(F^{(p)} / F_{i}\right) /\left(T / F_{i}\right)$. We remark that $T^{(1)}$ $\neq T^{(2)}$ implies that the closures in $G^{(p)}$ of $T_{1}, T_{2}$, respectively, are also distinct provided that $T^{(1)}$ and $T^{(2)}$ have finite indices under $F^{(p)}$. 
It is relatively easy to determine the exact number of regular fields $K$ with prescribed Galois group of small order. We want to discuss briefly the simplest case. Suppose that $\mu=1$, that is, $q-1=p \cdot r,(r, p)=1$. Then $A_{2}$ is a non-abelian extension of degree $p^{4}$ over $k$. Its Galois group is given by the relations

$$
\Sigma^{p^{2}}=\Gamma^{p^{2}}=1, \quad \Gamma^{-1} \Sigma \Gamma=\Sigma^{1+p}
$$

Now let $G$ be an arbitrary group of order $p^{3}$. Since the commutative cases are already settled by local class field theory, we investigate the non-abelian cases. There are two distinct types of non-abelian groups of order $p^{3}$ :

( $\alpha) \sigma^{p^{2}}=\tau^{p}=1, \tau^{-1} \sigma \tau=\sigma^{1+p}$ and

( $\beta$ ) $\sigma^{p}=\tau^{p}=\rho^{p}=1, \rho=\sigma \tau \sigma^{-1} \tau^{-1}, \rho^{-1} \sigma \rho=\sigma, \tau^{-1} \sigma \tau=\sigma, \rho^{-1} \tau \rho=\tau \sigma$ (cf. [2]). A simple computation yields that both groups are homomorphic maps of $G_{2}=\{\Sigma, \Gamma\}$. Hence every type has at least one realization as the Galois group $G(K)$ of a regular field $K \subset A_{2}$. Moreover, every $K$ which belongs to either type $(\alpha)$ or type $(\beta)$ must be contained in $A_{2}$. Since these fields $K$ are nonabelian extensions of $k$, they all must contain the field $A_{1}$. This field $A_{1}$ belongs [12.1] to the group $G_{2}^{*}=\left\{\Sigma \Gamma \Sigma^{-1} \Gamma^{p-1}\right\}$ of $G_{2}$. Since $K \supset A_{1}$, we also have $G / G^{*} \cong G_{2} / G_{2}^{*}$ where $G^{*}$ is defined in the same fashion as $G_{2}^{*}$. Moreover, $G_{2} / S^{\prime}(K)=G(K)$. Hence, by the homomorphism principle of group theory, $S^{\prime}(K) \subset G_{2}^{*}$. Thus, the fields $K$ are found among the cyclic extensions of degree $p$ over $A_{1}$. There are $p+1$ such extensions for $G_{2}^{*}$ has $p+1$ invariant subgroups of order $p$, as follows from the structure of $G_{2}$. Namely, $G_{2}^{*}=\left\{G_{2}^{\prime}\right.$; $\left.X^{p}, X \varepsilon G_{2}\right\}$ where $G_{2}^{\prime}$ is the commutator group of $G_{2}$ (cf. [12.1]). Consequently $G_{2}^{*}=\left\{\Sigma^{p}, \Gamma^{p}\right\}$ for $\Sigma^{-1} \Gamma^{-1} \Sigma \Gamma=\Gamma^{p} \varepsilon\left\{\Sigma^{p}, \Gamma^{p}\right\}$. Moreover $\Gamma^{-p} \Sigma \Gamma^{p}=\Sigma$ and $\Gamma^{-1} \Sigma^{p} \Gamma=\Sigma^{p}$.

Consider now the invariant subgroup $\left\{\Sigma^{p}\right\}$ of $G_{2}$. Its factor group $G_{2} /\left\{\Sigma^{p}\right\}$ is abelian and has type $\left(p, p^{2}\right)$. The associated field, belonging to $G_{2}^{\prime}$, is $U_{p^{2}} k\left(\lambda^{1 / p}\right)$.

The factor group $G_{2} /\left\{\Gamma^{p}\right\}$ is non-abelian. It has type $(\alpha)$.

The $p-1$ different subgroups given by $\left\{\Sigma^{i p} \Gamma^{j p}\right\}$ have factor groups which belong to type $(\beta)$. This is easily verified.

Combining these results we can state the following theorem.

TheOREM 5. If $p \| q-1$, then $k$ has exactly one regular extension of type $(\alpha)$ and exactly $(p-1)$ distinct regular extensions of type $(\beta)$.

The infinite discrete group $F^{(p)}$ can be considered as a generalization of the fuchsian groups of the classical theory of algebraic functions to $l$-adic number fields. The analogy is rather striking. We may interpret it as follows. The substitution $\tau \varepsilon F^{(p)}$ corresponds to the unramified extensions, that is, 
to the fundamental group of the classical case. The substitution $\sigma \varepsilon F^{(p)}$ corresponds to the substitution of signature $\lim _{i \rightarrow \infty} p^{-i}=p^{-\infty}$ around the given branch point of the underlying Riemann surface. Moreover, the commutator group $F^{(p) \prime}$ of $F^{(p)}$ is given by $\tau^{-1} \sigma \tau \sigma^{-1}=\sigma^{p^{\mu}}$ as is readily verified. The factor group $F^{(p)} / F^{(p) \prime}$ has type $\left(p^{\mu}, p^{\infty}\right)$. Using the ramification theory of infinite normal extensions of an $\mathfrak{l}$-adic number field, one sees that the subfield $L$ belonging to $F^{(p) \prime}$ is given as $k\left(\lambda^{1 / p^{\mu}}\right) U_{\infty}^{(p)}$ where $U_{\infty}^{(p)}$ denotes the join of all unramified extensions of degree $p^{i}(i=1,2, \cdots)$ over $k$ (cf. [6]). We remark that $F^{(p)} / F^{(p) \prime}=\left\{\tau, \sigma ; \sigma^{p^{\mu}}=1\right\}$ is the generalization of the Betti group belonging to a given branch point with signature $1 / p^{\mu}$.

The equivalent to the multiplicative functions belonging to a fuchsian group is given by the multiplicative group $N^{(p) *}$ of the universal field. The field $N^{(p)}$ contains all $p^{i+\mu}$ th roots of unity $\omega_{i}$ and all radicals $\lambda^{1 / p^{i}}=\lambda_{i}$, $i=1,2, \cdots$. Since $k$ is supposed to contain the $p$ th roots of unity, we have $U_{\infty}^{(p)}=k\left(\omega_{1}, \omega_{2}, \cdots, \omega_{i}, \cdots\right)$. Moreover, the construction of $N^{(p)}$ as $\sum A_{i}$ implies that $\lambda_{i} \varepsilon N^{(p)}$. Hence

$$
N^{(p)}=k\left(\omega_{1}, \cdots, \omega_{i}, \cdots ; \lambda_{1}, \cdots, \lambda_{i}, \cdots\right) .
$$

The groups $\left\{\omega_{i}, \lambda_{i}\right\}$ which are generated by the elements $\omega_{i}, \lambda_{i}$-for a fixed $\lambda$ in $k$-form a multiplicative subgroup $M^{(p)}$ of $N^{(p) *}$. The complete closure $\overline{N^{(p)}}$ of $N^{(p)}$ consists of all sums $\Phi \Psi \sum_{j=0}^{\infty} \Phi_{j} \Psi_{j}=P$ where $\Phi, \Phi_{j} \varepsilon\left\{\omega_{i}\right\}, \Psi, \Psi_{j} \varepsilon\left\{\lambda_{i}\right\}$ such that $V\left(\Psi_{j}\right) \rightarrow \infty$ where $V$ denotes the valuation of $N^{(p)} ; \dagger V\left(\Psi_{0}\right)=0$, $\Phi_{0} \neq 0$. Thus $N^{(p)}$ consists of all elements of $\overline{N^{(p)}}$ which are algebraic over $k$.

Now let $K$ be an arbitrary regular finite extension of $k$. The structure theory of complete fields yields that $K$ is complete and that it is uniquely determined by the root of unity $\omega(K)$ of highest order which is contained in $K$ and a prime element $\lambda(K)$ of $K$ :

$$
K=\left(\omega(K)^{a} \lambda(K)^{b} \sum_{j=0}^{\infty} \omega(K)^{c_{j} \lambda(K)^{j}}\right),
$$

the $c_{j}$ residues modulo the order of $\omega(K)$ (cf. [7, 11, 12.2]).

According to Theorem 2 we have $K \leqq A_{i}$, where $i$ is sufficiently large. Since $A_{i}=k\left(\lambda_{i}, \omega_{i}\right)$, we have

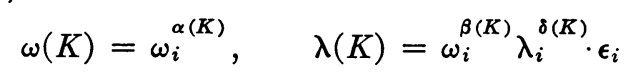

where $\epsilon_{i}$ is a unit of $A_{i}$ which is congruent to $1\left(\bmod \left(\lambda_{i}\right)\right)$.

Let $\{\rho\}$ be the Galois group of $A_{i} / K$. Then

$$
\lambda(K)=\lambda(K)^{\rho}=\left(\omega_{i}^{\beta(K)} \lambda_{i}^{\delta(K)} \epsilon_{i}\right)^{\rho}=\left(\omega_{i}^{\beta(K)} \lambda_{i}^{\delta(K)}\right)^{\rho \rho \rho} \epsilon_{i} .
$$

$\dagger[9]$. By Hensel's criterion applied to relatively complete fields. 
Hence $\left(\omega_{i}^{\beta_{(}(K)} \lambda_{i}^{\delta(K)}\right)^{\rho-1}=\epsilon_{i}^{1-\rho}$ for all $\rho$. The unit $\epsilon_{i}^{1-\rho}$ lies in $\left\{\epsilon_{i}\right\}$; moreover $\left\{\omega_{i}, \lambda_{i}\right\} \cap\left\{\epsilon_{i}\right\}=1$. Thus, $\epsilon_{i}^{1-\rho}=1$ for all $\rho$. For $\rho$ effects a multiplication of $\lambda_{i}$ by a root of unity according to the definition of $\lambda_{i}$ as a radical. Hence $\epsilon_{i} \varepsilon K$. Thus, $\lambda(K) \cdot \epsilon_{i}^{-1}=\lambda^{\prime}(K)=\omega_{i}^{\beta(K)} \lambda_{i}^{\delta(K)}$. In other words, the prime element $\lambda(K)$ of $K$ can be normalized in such a way that it lies in $\left\{\lambda_{i}, \omega_{i}\right\}$.

The group $F^{(p)}$ acts as operator group on $M^{(p)}$. Arranging the elements of $M^{(p)}$ as follows: $\left.\left\{\omega_{1}, \lambda_{1}\right\}, \cdots,\left\{\omega_{i}, \lambda_{i}\right\}\right\}$, and observing that $F^{(p)}$ is everywhere dense in $G^{(p)}$, it follows that this representation of $F^{(p)}$ as operator group is an isomorphic one. Let $S$ be an arbitrary normal subgroup of finite index under $F^{(p)}$. Denote by $M(S)$ the subgroup of all elements in $M^{(p)}$ which are left invariant by $S$. Since $S$ contains some $S_{i}$ belonging to a field $A_{i}$, it follows that $M(S) \subseteq\left\{\omega_{i}, \lambda_{i}\right\}$. We already noticed before that the field of $S$ is a uniquely determined subfield $K(S)$ of $A_{i}$. Hence we can apply the result concerning the normalization of the prime element of $K(S)$. It follows that $M(S)$ is equal to $\left\{\omega_{i}, \lambda_{i}\right\} \cap K(S)$. In order to determine the maximal root of unity $\omega(S)$ and the normalized prime element $\lambda(S)$ of $K(S)$ we proceed as follows. Since $M(S)$ is a finite group contained in $\left\{\omega_{i}, \lambda_{i}\right\}$, it has a minimal base of at most 2 elements. Let $\lambda(S)$ be an element of $M(S)$ such that $V(\lambda(S))$ is minimal. Then take for $\omega(S)$ an arbitrary other element of value 0 which has maximal order. The elements $\lambda(S), \omega(S)$, thus selected, obviously have the required properties. These results which are implied by the fact that we have to deal with (discrete) complete fields can be interpreted in the following fashion. The group $M^{(p)}$ is the minimal set of multiplicative functions over $k$ which suffices to describe all finite regular fields. Somehow the elements $\omega_{i}$ correspond to the unramified algebraic functions - obtained from normalized integrals of third kind-of the classical case. The $\lambda_{i}$ correspond to ramified algebraic functions with one prescribed pole. Of course, this analogy is rather superficial.

We now want to interpret the Galois groups $G_{i}$ of the fields $A_{i} / k$ in terms of certain factor groups of division algebras over $k$.

Let $D_{i}$ be the normal division algebra of degree $p^{i}$ over $k$ which is given by the following relations. The algebra $D_{i}$ is to consist of all finite sums $\sum_{j, k=1}^{p^{i}} a_{j, k} \Omega_{i}^{j} \Lambda_{i}^{k}$, where $a_{j, k}$ are elements of $k$ and $\Omega_{i}, \Lambda_{i}$ satisfy the relations

(i) $\Lambda_{i}^{p^{i}}=\lambda$,

(ii) $\Omega_{i}$ is a primitive $\left(q^{p^{i}}-1\right)$ st root of unity,

(iii) $\Lambda_{i}^{-1} \Omega_{i} \Lambda_{i}=\Omega_{i}^{q}$ (cf. [4]).

Let $\mathfrak{R}_{i}$ be the two-sided prime ideal of $D_{i}$ and $\left\{\mathrm{E}_{i}\right\}$ the totality of all units in $D_{i}$ which are congruent to $1\left(\bmod \mathfrak{R}_{i}\right)$. Obviously, the set $\left\{\mathbf{E}_{i}\right\}$ is a group.

Lemma 6. Every element of $\left\{\mathbf{E}_{i}\right\}$ is a pith power. 
Proof. Let $\mathrm{E}_{i}$ be an arbitrary element of $\left\{\mathrm{E}_{i}\right\}$. Then $\mathrm{E}_{i}=1+B_{i}$ where $V_{\varepsilon_{i}}\left(B_{i}\right)>0$. Consider the subfield $K\left(B_{i}\right)$ of $D_{i}$. It contains $\mathrm{E}_{i}$. Hence, by the proof of Lemma $1, \mathrm{E}_{i}=\mathrm{H}^{p^{i}}$ where $\mathrm{H}$ is a suitable unit which is congruent to 1 modulo the prime ideal of $k\left(B_{i}\right)$. Since $\mathrm{H} \varepsilon k\left(B_{i}\right) \subset D_{i}$, the assertion of the lemma is obvious.

THEOREM 6. The factor group $D_{i}^{*} / D_{i}^{* p^{i}}$ can be generated by two elements $\Lambda_{i}^{*}, \Omega_{i}^{*}$ satisfying the relations

$$
\Lambda_{i}^{* p^{i}}=\Omega_{i}^{* p^{i}}=1, \quad \Lambda_{i}^{*-1} \Omega_{i}^{*} \Lambda_{i}^{*}=\Omega_{i}^{* q} .
$$

Proof. We first remark that the group $D_{i}^{*_{p i}}$ which is generated by the $p^{i}$ th powers of all nonzero elements in $D_{i}^{*}$ is an invariant subgroup of the multiplicative group $D_{i}^{*}$. The group $D_{i}^{* p^{i}}$ contains the unit group $\left\{\mathrm{E}_{i}\right\}$, by Lemma 6. Thus, $\Lambda_{i}$ and $\Omega_{i}$ can be considered as representatives of $D_{i}^{*} / D_{i}^{* p^{i}}$. The defining relations of $D_{i}$ and the fact that $\left\{\mathrm{E}_{i}\right\}$ is an invariant subgroup of $D_{i}^{*}$ imply that every class of $D_{i}^{*} / D_{i}^{* p^{i}}$ can be represented as $\Lambda_{i} \alpha_{i} \Omega_{i}{ }^{\beta_{i}} D_{i}^{* p^{i}}$ where $0 \leqq \alpha_{i}, \beta_{i}<p^{i}$. Hence $\Lambda_{i}^{*}=\Lambda_{i}\left(\bmod D_{i}^{* p^{i}}\right)$ and $\Omega_{i}^{*}=\Omega_{i}\left(\bmod D_{i}^{* p^{i}}\right)$ have the required properties.

We can suppose that the Galois group $G_{i}$ of $A_{i} / k$ is given by the relations $\sigma_{i}^{p^{i}}=\tau_{i}^{p^{i}}=1, \tau_{i}^{-1} \sigma_{i} \tau_{i}=\sigma_{i}^{q}$. Namely, $q-1$ is exactly divisible by $p^{\mu}$.

THEOREM 7. The Galois group $G_{i}$ of $A_{i} / k$ is isomorphic with the factor group $D_{i}^{*} / D_{i}^{* p^{i}}$ of $D_{i}$.

Proof. The asserted isomorphism is an immediate consequence of Theorem 6 and Lemma 4 . We let correspond $\sigma_{i} \rightarrow \Omega_{i}^{*}, \tau_{i} \rightarrow \Lambda_{i}^{*}$.

The group $D_{i}^{* p^{i}}$ can be considered as the generalization of the norm groups appearing in local class field theory. We can obtain $D_{i}^{* p^{i}}$ by the following construction. Consider the norm group $N_{i} A_{i}^{*}=k^{* p^{i}}$ which belongs to the field $A_{i} / k$. Let $N_{i}$ denote the reduced norm of $D_{i}$ over $k$. Then $D_{i}^{*}{ }^{*}{ }^{i}$ is the subgroup $H$ of maximal index in $D_{i}^{*}$ such that $\mathcal{N}_{i} H=N_{i} A_{i}^{*}$. This follows immediately if one observes that $\mathcal{N}_{i} D_{i}^{*}=k^{*}$, and that $\Lambda_{i}, \Omega_{i},\left\{\mathbf{E}_{i}\right\}$ form a base for all elements of $D_{i}^{*}$. The requirement $\mathcal{N}_{i} H=N_{i} A_{i}^{*}$ implies certain congruence conditions for the exponents occurring in the multiplicative representation of the elements in $H$ in terms of the base $\Lambda_{i}, \Omega_{i},\left\{\mathrm{E}_{i}\right\}$. Since $\mathcal{N}_{i}$ is an abelian multiplicative function on $D_{i}^{*}$, it follows that $H$ is a normal subgroup of $D_{i}^{*}$.

Now let $D_{i}^{* \prime}$ be the maximal subgroup of $D_{i}^{*}$ such that $\mathcal{N}_{i} D_{i}^{* \prime}=N_{i} A_{i}^{*}$. Using the homomorphism principle on groups we get

$$
\left[D_{i}^{*}: D_{i}^{* \prime}\right]=\left[D_{i}^{*} / D_{i}^{* p^{i}}: D_{i}^{* \prime} / D_{i}^{* p^{i}}\right]=\left[k^{*}: N_{i} A_{i}^{*}\right] .
$$

The last index is equal to $\left[G_{i}: G_{i}{ }^{\prime}\right]$, where $G_{i}{ }^{\prime}$ denotes the commutator group 
of $G_{i}$ (cf. $\left.[1,8,10]\right)$. Hence $D_{i}^{* \prime} / D_{i}^{* p^{i}}$ is the commutator group of $D_{i}^{*} / G_{i}^{* p^{i}}$, for $D_{i}^{*} / D_{i}^{* p^{i}} \cong G_{i}$ by Theorem 7 . Thus, $D_{i}^{* \prime} / D_{i}^{*}{ }^{{ }^{i}} \cong G_{i}{ }^{\prime}$. The latter group is a cyclic group which is generated by the commutator $\tau_{i}^{-1} \sigma_{i} \tau_{i} \sigma_{i}^{-1}=\sigma_{i}^{q-1}$. Namely, the structure of $A_{i} / k$ implies that $A_{i}=U_{p^{i}}\left(\lambda^{1 / p^{\mu}}\right)\left(\lambda^{1 / p^{\mu}}\right)$ is a cyclic extension of degree $p^{i-\mu}$ over $U_{p^{i} k}\left(\lambda^{1 / p^{\mu}}\right)$. The latter field $A_{i}^{\prime}$ obviously belongs to $G_{i}{ }^{\prime}$ according to the local class field theory. We have

$$
\begin{aligned}
{\left[D_{i}^{*}: D_{i}^{* p^{i}}\right] } & =\left[\mathcal{N}_{i} D_{i}^{*}: \mathcal{N}_{i} D_{i}^{* p^{i}}\right]\left[X_{1}: Y_{1}\right], \\
p^{2 i} & =\left[k^{*}: N_{i} A_{i}^{*}\right]\left[X_{1}: Y_{1}\right]=p^{i+\mu} p^{i-\mu}, \quad \text { if } i>\mu .
\end{aligned}
$$

Here $X_{1}$ denotes the group of elements in $D_{i}^{*}$ whose reduced norms are equal to 1 and similarly, $Y_{1}$ the appropriate subgroup of $D_{i}^{* p^{i}}$. These statements yield that $D_{i}^{* \prime} / D^{p^{i}}$ is represented by a root of unity. For this we only have to take into account the structure of $D_{i}^{*}$ and the multiplicative properties of $\mathcal{N}_{i}$. It is therefore not so astounding that the Galois groups $G_{i}$ can be described by factor groups of $D_{i}^{*}$. The algebra $D_{i}$ contains the $\left(q^{p^{i}}-1\right)$ st roots of unity and hence the $p^{\mu+i}$ th roots of unity, for the maximal unramified subfield $U^{p^{i}}$ of $D_{i}$ has relative degree $p^{i}$ over $k$. Consequently $U_{p^{i}}$ must be given by $\omega^{1 / p^{i}}$, where $\omega$ is a primitive $(q-1)$ st root of unity in $k$, for we supposed $q-1 \equiv 0(p)$.

The field $A_{i}{ }^{\prime}$ can be obtained as follows. Consider the group $D_{i}^{*} / D_{i}^{* p^{i}}$ and reduce it modulo the commutator group $D_{i}^{* \prime} / D_{i}^{* p^{i}}$. Then $\Lambda_{i}^{* p^{\mu}}=\lambda^{*}$ and $\Omega_{i}^{*}{ }^{*}{ }^{i}=\omega^{*}$. Consider $\lambda^{*}$ and $\omega^{*}$ as the elements $\lambda, \omega$ in $k$. Then the solutions $x_{i}, y_{i}$ of $x_{i}^{p \mu}=\lambda, y_{i}^{p^{i}}=\omega$ define the field $A_{i}{ }^{\prime}$.

In order to obtain $A_{i}$ itself, we associate to $D_{i}^{*} / D_{i}^{*} p^{i}$ the group $\tilde{A}_{i}$ given by the relations $X_{i}^{p^{i}}=\lambda, Y_{i}^{p^{i}}=\omega$. Then $A_{i}=k\left(X_{i}, Y_{i}\right)$. This is to a certain extent the generalization of the theory of radical fields to normal regular extensions.

Suppose now that $K$ is an arbitrary regular normal extension of $k$. Then $K \subseteq A_{i}$ for some $A_{i}$ by Theorem 2 . To the field $K$ there corresponds a uniquely determined invariant subgroup $\bar{H}$ of $G_{i}$ such that $G(K, k) \cong G_{i} / \bar{H}$. The local class field theory yields that $G(K, k) / G^{*}(K, k)^{\prime} \cong k^{*} / N_{K} K^{*}$, where $G(K, k)^{\prime}$ denotes the commutator group of $G(K, k)$ and $N_{K} K^{*}$ stands for the norm class group of $K$ with respect to $k$. Theorem 7 implies the existence of a unique invariant subgroup $H$ of $D_{i}^{*}$ such that $D_{i}^{*} / H \cong G(K, k)$.

THEOREM 8. Let $K$ be an arbitrary normal regular extension of $k$ such that $K \subseteq A_{i}$, then $\left[k^{*}: \mathcal{N}_{i} H\right]=\left[k^{*}: N_{K} K^{*}\right]$.

Proof. The first isomorphism theorem of group theory yields that there exists an invariant subgroup $\bar{R}$ of $G_{i}$ such that $G_{i} / \bar{R} \cong G(K, k) / G(K, k)^{\prime}$. Hence $\bar{R} \supseteqq G_{i}{ }^{\prime}$ by the properties of the commutator group $G_{i}{ }^{\prime}$. Trans- 
ferring $\bar{R}$ to $D_{i}^{*}$, we obtain an invariant subgroup $R$ of $D_{i}^{*}$ for which $D_{i}^{*} / R \cong G(K, k) / G(K, k)^{\prime} \cong k^{*} / N_{K} K^{*} ; R \supseteqq D_{i}^{* \prime}$. Next consider the index $\left[D_{i}^{*}: H\right]$. Applying the multiplicative mapping $N_{i}$ to $D_{i}^{*}$ and $H$, we get

$$
\left[D_{i}^{*}: H\right]=\left[\mathcal{N}_{i} D_{i}^{*}: \mathcal{N}_{i} H\right]\left[D_{i}^{* \prime}: D_{i}^{* \prime} \cap H\right]=\left[k^{*}: \mathcal{N}_{i} H\right]\left[D_{i}^{* \prime}: D_{i}^{* \prime} \cap H\right] .
$$

The second isomorphism theorem of group theory yields

$$
D_{i}^{* \prime} / D_{i}^{* \prime} \cap H \cong D_{i}^{*} \cup H / H .
$$

Consequently, $\left[k^{*}: \mathcal{N}_{i} H\right]=\left[D_{i}^{*}: H \cup D_{i}^{* \prime}\right]$. Namely, the inclusion $D_{i}^{*} \supseteqq H \cup D_{i}^{* \prime}$ $\supseteqq H$ implies

$$
\left[D_{i}^{* \prime} \cup H: H\right]=\left[D_{i}^{*}: H\right]\left[D_{i}^{*}: D_{i}^{* \prime} \cup H\right]^{-1} .
$$

Thus $\left[D_{i}^{*}: H\right]=\left[k^{*}: \mathcal{N}_{i} H\right]\left[D_{i}^{*}: H\right]\left[D_{i}^{*}: D_{i}^{* \prime} \mathbf{u} H\right]^{-1}$. Since $D_{i}^{*} / R$ is an abelian group, we have $R \supseteqq D_{i}^{* \prime}$, by the properties of the commutator group $D_{i}^{* \prime}$. Hence $R \supseteqq D_{i}^{* \prime} \mathbf{v} H$. But also $D_{i}^{* \prime} \cup H \leqq R$ for $R / H$ is the commutator group of $D_{i}^{*} / H$. Consequently, $D_{i}^{* \prime} \mathbf{u} H=R$.

Application of $N_{i}$ to the groups $D_{i}^{*}$ and $D_{i}^{*} \mathrm{v} H$ yields

$$
\begin{aligned}
{\left[D_{i}^{*}: D_{i}^{* \prime} \cup H\right] } & =\left[\mathcal{N}_{i} D_{i}^{*}: \mathcal{N}_{i} D_{i}^{* \prime} \cap H\right]\left[D_{i}^{* \prime}: D_{i}^{* \prime} \cup\left(H \cap D_{i}^{* \prime}\right)\right] \\
& =\left[k^{*}: \mathcal{N}_{i} H\right] .
\end{aligned}
$$

Finally, $\left[k^{*}: N_{K} K^{*}\right]=\left[k^{*}: \mathcal{N}_{i} H\right]$ for $D_{i}^{*} / R \cong G(K, k) / G(K, k)^{\prime}$.

Finally we want to indicate briefly that the theory of regular normal extensions $K / k$ cannot be developed with respect to a fixed division algebra $D_{i}$ of degree $p^{i}$ over $k$. It would be natural to require that every Galois group $G$ is a homomorphic map of $D_{i}^{*}$. In analogy to the theory of abelian fields (which can be described by subgroups of $k^{*}$ ) we would require that all units $\left\{\mathrm{E}_{i}\right\}$ of $D_{i}$ are contained in the subgroups $H$ of $D_{i}^{*}$ belonging to the arbitrarily given field $K / k$. Namely, the set of units $\mathrm{E}_{t}^{(j)} \equiv\left(\left(\Lambda_{i}\right)^{j}\right)$ form an invariant subgroup of the totality of all units in $D_{i}$. The index of this subgroup turns out to be equal to $q^{p^{i} j-1}\left(q^{p^{i}}-1\right)$. Thus $p^{\mu p^{i}} \| q^{p^{i} j-1}\left(q^{p^{i}}-1\right)$ for $D_{i}$ contains no units of higher $p$-order than $p^{\mu p^{i}}$, as follows from the algebraic theory of splitting fields of $D_{i}$. Hence the class groups $H$ which supposedly can be associated to the fields $K / k$ must contain $\mathrm{E}_{i}$. Consequently, any such invariant group $H$ of $D_{i}$ must contain $\left\{\Omega_{i}^{m}\right\} \cup\left\{\Lambda_{i}^{s}\right\}$ for suitable chosen integers $m$, s. Since $D_{i}$ contains only roots of unity of bounded $p$-order, the possible exponents, are bounded, say $m \leqq m_{0}$. If $m>m_{0}$, then $\left\{\Omega_{i}^{m}\right\} \mathbf{u}\left\{\Lambda_{i}^{s}\right\}=\left\{\Omega_{i}^{m_{0}}\right\} \mathbf{u}\left\{\Lambda_{i}^{s}\right\}$. We have a similar fact in local class field theory. There the ramification exponents $e(K)$ of regular fields are bounded by $p^{\mu}$. The reason being that the ground field $k$ does not contain sufficiently may roots of unity. Such roots of unity always are needed to describe the class groups of ramified abelian extensions. In 
other words, in the abelian case the regular extensions are made up by a ramified extension of degree $p^{\mu}$ and an unramified extension of arbitrarily high degree (cf. [10]). A similar situation prevails in the general case. Having fixed an algebra $D_{i}$, we only can describe by means of $D_{i}$ such regular fields $K$ which contain a normal subfield whose ramification degree is not greater than $p^{\mu p^{i}}$. One also could arrive at this result by purely group theoretical analysis. The structure of $D_{i}^{*} /\left\{\Omega_{i}^{m}\right\} \mathbf{u}\left\{\Lambda_{i}^{s}\right\}$ can easily be determined. One observes that not all $G_{i}$ can be homomorphic maps of some suitable $D_{i}^{*} /\left\{\Omega_{i}^{m}\right\} \mathbf{u}\left\{\Lambda_{i}^{s}\right\}$. Hence, by virtue of Theorem 2 , not every $G$ can be described as a homomorphic map of $D_{i}^{*}$. These considerations indicate why the totality of all algebras $D_{i}$ is required for our generalization of local class field theory.

\section{BIBLIOGRAPHY}

1. Y. Akizuki, Eine homomorphe Zuordnung der Elemente der galoisschen Gruppe zu den Elementen einer Untergruppe der Normklassengruppe, Mathematische Annalen, vol. 112 (1936).

2. W. Burnside, Theory of Groups of Finite Order.

3. C. Chevalley, La théorie du symbole de restes normiques, Journal für die reine und angewandte Mathematik, vol. 169 (1933).

4. H. Hasse, Über p-adische Schiefkörper und ihre Bedeutung für die Arithmetik hyperkumplexer Zahlsysteme, Mathematische Annalen, vol. 104 (1930).

5. K. Hensel, (1) Allgemeine Theorie der Kongruenzklassengruppen und ihrer Invarianten in algebraischen Zahlkörpern, Journal für die reine und angewandte Mathematik, vol. 147 (1917); (2) Zahlentheorie, Berlin and Leipzig.

6. J. Herbrand, Théorie arithmétique des corps de nombres de degré infini, II. Extensions algébriques de degré infini, Mathematische Annalen, vol. 108 (1933).

7. S. MacLane, Subfields and automorphisms of p-adic fields, Annals of Mathematics, (2), vol. 40 (1939).

8. T. Nakayama, Über die Beziehungen zwischen den Faktorensystemen und der Normklassengruppe eines galoisschen Erweiterungskörpers, Mathematische Annalen, vol. 112 (1936).

9. A. Ostrowski, Untersuchungen zur arithmetischen Theorie der Körper, Mathematische Zeitschrift, vol. 39 (1935).

10. F. K. Schmidt, Zur Klassenkörpertheorie im Kleinen, Journal für die reine und angewandte Mathematik, vol. 162 (1930).

11. O. Teichmüller, Diskret bewertete perfekte Körper mit unvollkommenem Restklassenkörper, Journal für die reine und angewandte Mathematik, vol. 176 (1936).

12. E. Witt, (1) Konstruktion von galoisschen Körpern der Charakteristik p mit gegebener Gruppe der Ordnung $p^{n}$, Journal für die reine und angewandte Mathematik, vol. 174 (1936); (2) Zyklische Körper und Algebren der Charakteristik p vom Grade $p^{n}$, Journal für die reine und angewandte Mathematik, vol. 176 (1936).

University of Chicago, ChicAgo, Ill. 